\title{
Cyclic Scheduling of Single-Arm Cluster Tools with Multiple Wafer Types: A Case Study
}

\author{
Xin LI and Jiongjiong TIAN ${ }^{1}$ \\ College of Management, Shenzhen University, China
}

\begin{abstract}
Cluster tools are widely used in the industrial production process, such as semiconductor wafers, printing circuit board and other fields. In this paper, the scheduling problem of cluster tools with multi-type wafers is studied, including robot handling jobs sequencing and wafer processing sequencing. Based on the detailed analysis of the operations in the problem, there are the processing time window constraints, robot available constraints, processing capacity constraints. Then, due to the complexity in the general conditions, a case with four different wafers (including one for each type in a cycle) is considered. The applied model was solved by the third-party library Gurobi in PyCharm.
\end{abstract}

Keywords. Single-arm cluster tool, multiple types of wafer, shared processing chambers, time window, circle scheduling.

\section{Introduction}

Cluster tools usually composed of four modules, i.e. handling, processing, alignment and loading. The handling module is generally composed of a robot arm, and the processing module is composed of several processing chambers. The alignment module and loading module are generally in the same place. Figure 1 shows a single arm cluster tools. In modern industrial production, a cluster tool is widely used in the manufacturing process of electronic products such as semiconductor wafers, printing circuit board and other fields due to its high efficiency, automation and convenient operation.

Because the increasing diversification of customer demands and the increase of the wafer size, the multiple wafer types production is becoming the mainstream trend in the production process of electronic products. In addition, in order to better improve the production efficiency of cluster tools and reduce the cost of equipment procurement, manufacturers often arrange multiple types of wafer in a cluster tool at the same time. For example, in a wafer production lot, the four wafers are produced in a ratio of $3: 2: 3: 2$, and each lot is produced in accordance with this ratio. This is the minimum workpiece set [1]. When multiple wafers are produced in a cluster tool at the same time, some processing chambers are often used by many kinds of wafer. These chambers are called as shared processing chambers. when multiple wafers are produced in this single processing chamber, the scheduling problem is arose.

${ }^{1}$ Corresponding Author, Jiongjiong TIAN, College of Management, Shenzhen University, Shenzhen, Guangdong, 518060, China; E-mail: 935522596@qq.com. 
In addition, when a wafer is processed in processing chambers, it usually needs to wait in the processing chamber for some time. Since there is no buffer module in the cluster tool, the wafer must be unloaded from the processing chamber by the robot arm after it is processed. It means that the wafer cannot stay in the processing chamber beyond its upper bound, which is the processing time window.

In order to improve the productivity of cluster tools, in the process of wafer production tend to periodically production. In each production cycle has a batch of wafer enter the cluster tools for production. At the same time, another batch of wafer out of production system. In each of the production cycle, the robot arm performs same handling operations. The time interval between wafer production batches is known as cycle time or production beat [2]. The production process of cluster equipment can be expressed as follows. A batch of wafer in the loading station is corrected by the robot arm, then one by one enter the cluster tools, according to their preset processing procedures, the robot arm carry them one by one to the processing chamber. When the last wafer of a batch is moved to the unloading station, the wafer production batch is finished.

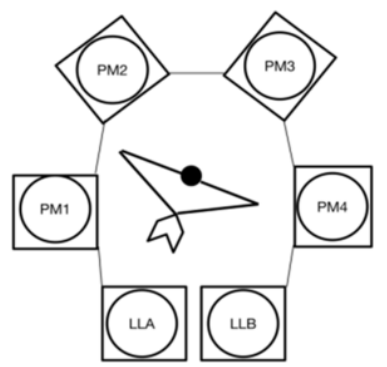

Figure 1. Single-arm cluster tool

\section{Literature Review}

Scholars have done some research on the cluster tools for processing multiple types wafer at the same time and published some results. Lee et al.[3] studied the scheduling problem of wafers of various types and solved the problem by deriving mathematical expressions. Zhou et al.[4] studied the scheduling problem of dual-arm cluster tools with re-entrant and parallel cavity constraints for multi-wafer types, and developed a scheduling method with optimization search as the core to solve this problem.

Although the above scholars have done some research on multi-wafer types, they have not considered the constraints of the processing time window. In the process of wafer production, because there are usually no storage modules, the wafer must be moved by a robot arm within a given time window after processed. This is a prominent constraint in wafer production. Wang et al.[5] studied the scheduling problem of multi-wafer types in cluster tools with residency time constraints. They developed multiplex backward sequence strategy and virtual module technology to optimize the research problem. However, they didn't take into account situations beyond the backward sequence strategy. Liu et al.[6] added the processing time window constraint to the multi-type wafer scheduling for cluster devices. But they only considered the number of wagers, not the type. It will not meet the demands of users for multiple wafers. 
Lei et al.[1] considered the processing time window constraints and multi-type of wafer production constraints. However, in this paper, the moving time of the robot arm is closely related to its movement between the various processing chambers. It makes the moving time of the robot arm between the two most distant processing chambers too long. Therefore, this will reduce the production efficiency of the cluster tools. Considering the shortcomings of the above problems, a new research problem is proposed in this paper. It is scheduling of multi-type wafers in a single-arm cluster tool with processing time window constraints and shared processing chamber constraints. In each wafer production cycle, each type of production process is not the same. Some different types of wafers will use the same processing chamber. Because multiple wafers will be produced in the same processing chamber, the scheduling problem is that which is produced first and which is produced later. It forms the constraint of shared processing chamber. In addition, this paper also considers that the processing time window of each wafer in each process is not consistent, which is more close to the actual wafer production process.

\section{Description of the Problem}

The cluster tool in this paper is composed of a robot arm, six processing chambers and two loading stations. Suppose $\mathrm{W}(\mathrm{W}>=2)$ different types of wafer are produced in a wafer production cycle, and the types of wafers are labeled $1,2,3 \ldots \mathrm{W}$. The different types of wafers are moved from the loading station enter the cluster tool by the robot arm in turn according to the respective processing procedures of each wafer. When all the processes of all wafers are completed, they are moved to the unloading station and then left the cluster tool. In a cluster tool, the process of different types of wafers is different, and the processing time in each process is different. A wafer must be processed at a predetermined time in each of its processing chambers. Since there are no buffer modules in the cluster tools, it must be moved by a robot to the next chamber for processing within a given time window [7]. Due to the unique characteristics of the cluster tool, only one wafer can be loaded or unloaded by robot arm and only one wafer can be processed by processing chamber at a same time. In addition, this paper also studies a circle scheduling problem. In each wafer production cycle, $\mathrm{W}$ types of wafers enter the cluster tool for processing, and W types of wafers leave the cluster tool after processed. Feasible scheduling can be achieved only when the cluster tools meets the following conditions e.g. processing time window constraints, robot available constraints, processing capacity constrains. Table 1 and table 2 describe the definitions of parameters and variables

Table 1. Definition of symbols

\begin{tabular}{cc}
\hline $\mathbf{W}$ & type of wafer \\
\hline $\left.\mathrm{a}_{\mathrm{wi}}, \mathrm{b}_{\mathrm{wi}}\right]$ & The i-th processing procedure of the w-th wafer \\
$\operatorname{move}(\mathrm{w}, \mathrm{i})$ & $\begin{array}{c}\text { The processing time window of type w-wafer in i-processing chamber } \\
\text { The process of moving a w-type wafer from its i-processing chamber to } \\
\text { the next processing chamber by the robot arm } \\
\text { the time it takes a robot to unload a wafer } \\
\text { the time it takes a robot to load a wafer }\end{array}$ \\
\end{tabular}


Table 2. Definition of variables

\begin{tabular}{|c|c|}
\hline $\mathrm{p}_{\mathrm{wi}}$ & $\begin{array}{l}\text { The time required for w-type wafer to be processed at i-processi } \\
\text { chamber }\end{array}$ \\
\hline $\mathrm{t}_{\mathrm{wi}}$ & $\begin{array}{l}\text { The start time of robot arm carries w-wafer from i-processing cl } \\
\text { to the next processing chamber in a wafer lot }\end{array}$ \\
\hline $\mathrm{y}_{w i, w i}=\left\{\begin{array}{l}1, t_{w i j} \leq t_{w i}{ }_{w} \\
0, t_{w i j} \\
\geq t_{w i}{ }_{w}\end{array}\right.$ & $\begin{array}{l}t_{w i} \leq t_{w^{\prime} i^{\prime}}: \operatorname{move}(w, i) \text { starts earlier than move(w',i'), } y_{w i, w^{\prime} i^{\prime}}=1 \\
t_{w i} \geq t_{w^{\prime} i^{\prime}}: \operatorname{move}\left(w^{\prime}, i^{\prime}\right) \text { starts earlier than move(w,i), } y_{w i, w^{\prime} i^{\prime}}=0\end{array}$ \\
\hline
\end{tabular}

The following figure shows the circle scheduling of wafer production. The cluster tool consists of six processing chambers, a robot arm, and two loading stations. Including one for each type in a cycle. At the start of the wafer production lot, the robot arm unloaded the third wafer at PM4 and moved it to PM5 for loading. Then it unloaded the second wafer at PM2 and moved it to PM3 for loading. Then it unloaded the first wafer at the loading station and waited for a period of time, and moved it to PM1 for loading. In this order, a wafer production cycle is completed until the robot arm unloads the fourth wafer from PM5 and moves it to PM6 for loading, and then drives to PM4 to wait.

\section{Establishment of Constraint Conditions}

\subsection{Constraints on Processing Time Window}

Refers to the wafer processing time window in the processing chamber of actual processing time must be within a specific time interval[8]. If the processing time is less than lower bounds, it will make the processing chamber of wafer processing tasks cannot be completed. If the processing time is greater than upper bounds, it will damage the wafer. In this paper, there are two situations in the initial processing stage of wafers. First, the robot arm loads the wafer into the processing chamber, which is the loading and unloading of wafers are in a production cycle. Second, the robot arm removes the wafer from the processing chamber. It means that the wafer has already been loaded into the processing chamber during the previous wafer production cycle, which is the loading and unloading of the wafer are not in the same production cycle.

When the i processing chamber of the $\mathrm{w}$ wafer at the beginning of the production cycle is idle, it loads $w$-type wafer at the $t_{w(i-1)}+\alpha+\theta+\beta$ moment to the $i$ processing chamber, At this time, the robot arm unloads it from the processing chamber at $t_{w i}$ moment. Therefore, the processing time of $w$-type wafer in i processing chamber is $p_{w i}$, it is equal to $t_{w i}-\left(t_{w(i-1)}+\alpha+\beta+\theta\right)$. Therefore, the processing time window can be represented as $a_{w i} \leq t_{w i}-\left(t_{w(i-1)}+\alpha+\beta+\theta\right) \leq b_{w i}$.

When the i processing chamber of the $w$ wafer is processing at the beginning of the production cycle, the robot arm loads the w-type wafer into the i processing chamber at the $t_{\mathrm{w}(\mathrm{i}-1)}+\alpha+\theta+\beta-\mathrm{T}$ moment. At this time, the robot arm unloads it from the processing chamber at $t_{w i}$ moment. Therefore, the processing time of the w-type wafer 
in i processing chamber is $p_{w i}$, it is equal to $t_{w i}+T-\left(t_{w(i-1)}+\alpha+\beta+\theta\right)$. In this situation, the processing time window can be represented as $t_{\mathrm{wi}}+\mathrm{T}-\left(\mathrm{t}_{\mathrm{w}(\mathrm{i}-1) \mathrm{j}}+\alpha+\beta\right.$ $+\theta) \leq \mathrm{b}_{\mathrm{wi}}$. The following constraints are obtained by introducing the $0-1$ variable $\mathrm{y}_{\mathrm{w}(\mathrm{i}-1), \mathrm{wi}}$.

$$
\begin{aligned}
& \mathrm{a}_{\mathrm{wi}}+\alpha+\beta+\theta-\mathrm{M}\left(1-\mathrm{y}_{\mathrm{w}(\mathrm{i}-1), \mathrm{wi}}\right) \leq \mathrm{t}_{\mathrm{wi}}-\mathrm{t}_{\mathrm{w}(\mathrm{i}-1)} \\
& \mathrm{t}_{\mathrm{wi}}-\mathrm{t}_{\mathrm{w}(\mathrm{i}-1)} \leq \mathrm{b}_{\mathrm{wi}}+\alpha+\beta+\theta+\mathrm{M}\left(1-\mathrm{y}_{\mathrm{w}(\mathrm{i}-1), \mathrm{wi}}\right) \\
& \mathrm{a}_{\mathrm{wi}}+\alpha+\beta+\theta-\mathrm{M}\left(1-\mathrm{y}_{\mathrm{w}(\mathrm{i}-1), \mathrm{wi}}\right) \leq \mathrm{T}+\mathrm{t}_{\mathrm{wi}}-\mathrm{t}_{\mathrm{w}(\mathrm{i}-1)} \\
& \mathrm{T}+\mathrm{t}_{\mathrm{wi}}-\mathrm{t}_{\mathrm{w}(\mathrm{i}-1)} \leq \mathrm{b}_{\mathrm{wi}}+\alpha+\beta+\theta+\mathrm{M}\left(1-\mathrm{y}_{\mathrm{w}(\mathrm{i}-1), \mathrm{wi}}\right)
\end{aligned}
$$

\subsection{Constraints on the Handling Capacity of Robot Arms}

This paper studies single-arm cluster tool, only one robot arm in the wafer production system. Since the robot arm can carry only one wafer in the same time, there are only two different orders of handling operations. First, the handling operation move (wi) earlier than move(w'i'), it is equal to $t_{w i}<t_{w^{\prime} i^{\prime}}$. Second, the handling operation move(w'i') earlier than move(wi), it is equal to $t_{w i}>t_{w^{\prime} i^{\prime}}$.

The first case, $t_{w_{i}}<t_{w^{\prime} i^{\prime}}$. At $t_{w(i-1)}+\alpha+\theta+\beta$ moment, the robot arm loads the w-type wafer into the $i$ processing chamber. Then the robot arm carries the wafer from the $i$ processing chamber to the $(i+1)$ processing chamber at $t_{w i}$ moment. At $t_{w i}+\alpha+$ $\theta+\beta$ moment, the robot arm from the (i+1) processing chamber to i' processing chamber for handling operation move ( $w$ 'i'). Therefore, the handling capacity constraint of the robot arm hand can be expressed as $t_{\mathrm{wi}}+\alpha+\theta+\beta+\theta \leq \mathrm{t}_{\mathrm{w}^{\prime} \mathrm{i}^{\prime}}$.

The second case, $t_{\mathrm{w}^{\prime} \mathrm{i}^{\prime}}<\mathrm{t}_{\mathrm{wi}}$. At $\mathrm{t}_{\mathrm{w}^{\prime}\left(\mathrm{i}^{\prime}-1\right)}+\alpha+\theta+\beta$ moment, the robot arm loads the w'-type wafer to the $i$ ' processing chamber. Then the robot arm carries the wafer from the $i^{\prime}$ processing chamber to the $\left(i^{\prime}+1\right)$ processing chamber at $t_{w^{\prime} i^{\prime}}$ moment. At $\mathrm{t}_{\mathrm{w}^{\prime} \mathrm{i}^{\prime}}+\alpha+\theta+\beta$ moment, the robot arm from the (i'+1) processing chamber to $\mathrm{i}$ processing chamber for handling operation move (wi). Therefore, the handling capacity constraint of the robot arm can be expressed as $t_{w^{\prime} i^{\prime}}+\alpha+\theta+\beta+\theta \leq t_{w i}$.

The following constraints are obtained by introducing the $0-1$ variable .

$$
\begin{aligned}
& \mathrm{t}_{\mathrm{w}^{\prime i^{\prime}}}-\mathrm{t}_{\mathrm{wi}} \geq \alpha+\theta+\beta+\theta-\mathrm{M}\left(1-\mathrm{y}_{\mathrm{wi}, \mathrm{w}^{\prime} \mathrm{i}^{\prime}}\right) \\
& \mathrm{t}_{\mathrm{wi}}-\mathrm{t}_{\mathrm{w}^{\prime \mathrm{i}^{\prime}}} \geq \alpha+\theta+\beta+\theta-\mathrm{My}_{\mathrm{wi}, \mathrm{w}^{\prime} \mathrm{i}^{\prime}}
\end{aligned}
$$

\subsection{Constraint of Processing Cycle Time}

In the process of wafer processing, in order to improve production efficiency, the wafer processing process often set as periodic production. In the same production cycle, there is a batch of wafers will be unload by robot arm after the processing is completed. Meanwhile, another batch of wafers is loaded into the cluster tools by the robot arm. Those wafers are processed and handed by a fixed sequence. We suppose $t_{w i}$ as the time when the last wafer starts to be transported in a cycle. Since $T$ is the production cycle of the entire production activity, it is greater than $t_{w i}$. At the same time, the periodicity of 
production makes the robot arm does not carry the wafer to the position at the beginning of the production cycle. According to the above content, the following constraint can be obtained.

$$
\mathrm{T}>=\mathrm{t}_{\mathrm{wij}}+\alpha+\theta+\beta+\theta
$$

\subsection{Constraints of Sharing Processing Chambers}

Since PM5 is shared by the third wafer and the fourth wafer, the scheduling problem is more complicated. After the analysis of the scheduling problem and the $0-1$ variable is substituted, the table 3 is obtained.

Table 3. The $0-1$ variable for $\mathrm{Pm} 5$

\begin{tabular}{cccccc}
\hline $\mathbf{y}_{311,312}$ & $\mathbf{y}_{311,401}$ & $\mathbf{y}_{311,411}$ & $\mathbf{y}_{321,401}$ & $\mathbf{y}_{321,411}$ & $\mathbf{y}_{401,411}$ \\
\hline 1 & 1 & 1 & 1 & 1 & 1 \\
1 & 1 & 0 & 1 & 0 & 0 \\
0 & 0 & 0 & 1 & 1 & 1 \\
1 & 0 & 0 & 0 & 0 & 1 \\
\hline
\end{tabular}

Table 3 is obtained on the basis of analyzing that the processing sequence of the third and fourth wafers does not conflict in PM5. The introduction of the $0-1$ variable in the article can obtain the following constraints on the shared processing chamber PM5.

$$
\begin{aligned}
& y_{31,41} \leq y_{31,32} \\
& y_{31,41} \leq y_{31,40} \\
& y_{31,41} \leq y_{32,40} \\
& y_{31,41} \leq y_{32,41} \\
& y_{31,41} \leq y_{40,41} \\
& 1-y_{31,32} \leq y_{40,41} \\
& 1-y_{31,40} \leq y_{40,41} \\
& 1-y_{32,40} \leq y_{40,41} \\
& y_{32,41} \leq y_{40,41} \\
& 1-y_{31,32} \leq y_{32,40} \\
& y_{31,40} \leq y_{32,40}
\end{aligned}
$$




$$
\begin{aligned}
& y_{32,41} \leq y_{32,40} \\
& 1-y_{40,41} \leq y_{32,40} \\
& y_{31,40} \leq y_{31,32} \\
& 1-y_{32,40} \leq y_{31,32} \\
& 1-y_{32,41} \leq y_{31,32} \\
& 1-y_{40,41} \leq y_{31,32} \\
& y_{31,32}+y_{32,40}+y_{40,41} \leq y_{31,41}+2 \\
& y_{31,32}+y_{32,40}+y_{40,41} \leq y_{32,41}+2
\end{aligned}
$$

\section{Illustrative Example}

In this paper, the mixed integer programming model was coded in Python language and solved by Gurobi (V9.1.2) in the programming software PyCharm. The validity and practicability of the model are tested by examples below. The background of example selection is semiconductor wafer processing.

The instance [5] in the cluster type tool is made up of six processing chambers and two loading chamber, shown in figure 2 . The time of loading, unloading and moving between the processing chamber is $3 \mathrm{~s}, 6 \mathrm{~s}$ and $6 \mathrm{~s}$ respectively. A production batch wafer type is four, all types of wafer processing number is 1 . Among them, the procedures of each type wafer and the processing time window of each processing chamber is shown in table 4 and table 5.

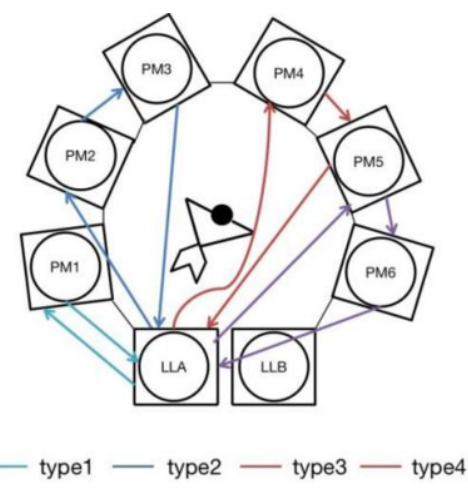

Figure 2. The case of a single arm cluster tool 
Table 4. The processing procedure of each wafer

\begin{tabular}{cc}
\hline Wafer type & Manufacturing procedure \\
\hline Type 1 & PM0 $\rightarrow$ PM1 $\rightarrow$ PM7 \\
Type 2 & PM0 $\rightarrow$ PM2 $\rightarrow$ PM3 $\rightarrow$ PM7 \\
Type 3 & PM0 $\rightarrow$ PM4 $\rightarrow$ PM5 $\rightarrow$ PM7 \\
Type 4 & PM0 $\rightarrow$ PM5 $\rightarrow$ PM6 $\rightarrow$ PM7 \\
\hline
\end{tabular}

Table 5. Processing time Windows of wafers in six processing chambers

\begin{tabular}{ccccc}
\hline $\mathbf{w}$ & $\mathbf{a}_{\mathbf{w 1}}$ & $\mathbf{b}_{\mathbf{w 1}}$ & $\mathbf{a}_{\mathbf{w} \mathbf{2}}$ & $\mathbf{b}_{\mathbf{w} \mathbf{2}}$ \\
\hline 1 & 142 & 158 & & 537 \\
2 & 480 & 507 & 516 & 353 \\
3 & 297 & 325 & 329 & 337 \\
4 & 329 & 353 & 311 & 3 \\
\hline
\end{tabular}

The model was coded and solved by PyCharm on the personal computer of MI1 chip. After running for $0.01 \mathrm{~s}$, the optimal production cycle time of the scheduling problem was $724 \mathrm{~s}$, indicating that the cluster device could process four different types of wafers in about 12 minutes. This shows the practicability and rapidity of the model. Among them, the scheduling process can be seen in the figure 3 .

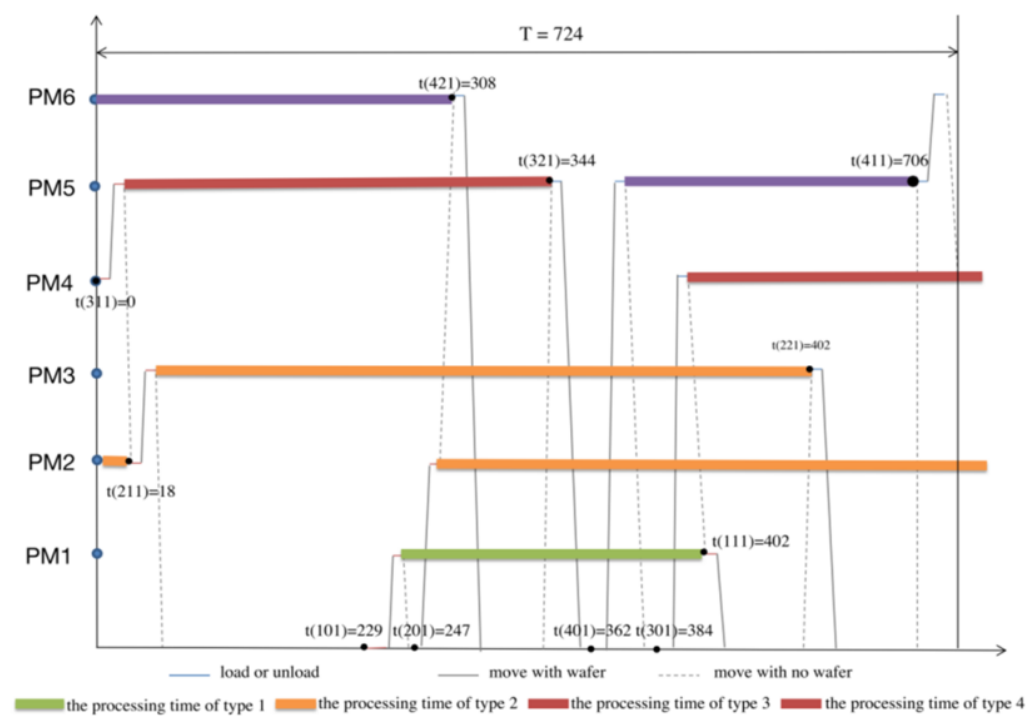

Figure 3. Cyclic scheduling

\section{Conclusion}

In this paper, a mathematical model for the scheduling problem is established by the mixed integer programming method, and the mixed integer programming model is verified by a production example of semiconductor wafer. The example result shows that the method and the model established by this method can obtain the optimal production 
cycle time of the scheduling problem and the start time of each robot arm handling operation in a reasonable time interval, which shows the method adopted in this paper can solve this kind of scheduling problem effectively and has high practicability and convenience. In the further research, a more general mixed integer programming model for this kind of problem will be constructed to solve the more general scheduling problem.

\section{References}

[1] Lei Weidong, Che Ada. Automatic Mixed-flow Production Line Scheduling Method with Time Window [J]. Industrial Engineering, 2011, 14(05): 110-115.

[2] Che A,Chu C. Multi-degree cyclic scheduling of a no-wait robotic cell with multiple robots [J. European Journal of Operation Research, 2009, 199( 1): 77-88.

[3] J. Lee and H. Kim. Makespan Analysis of Lot Switching Period in Cluster Tools [J] IEEE Transactions on Semiconductor Manufacturing, 2016, 29 (2)127-136.

[4] Zhou Binghai, Li Ming, Su Yi. Scheduling Method of Dual-arm Cluster Equipment with Parallel Cavity and Reentrant Constraints [J]. Journal of Engineering Science, 2016, 38(08): 1190-1195.

[5] J. Wang, C. Pan, H. Hu, L. Li and Y. Zhou. A Cyclic Scheduling Approach to Single-Arm Cluster Tools With Multiple Wafer Types and Residency Time Constraints [J] IEEE Transactions on Automation Science and Engineering, 2019,16 (3)1373-1386.

[6] Liu Mingxiang, Zhou Binghai. Scheduling Method of Cluster Equipment Group Based on Time Constraint Set [J]. Acta Automatica Sinica, 2012, 38(03): 479-485.

[7] Pan C R, Zhou M C, Qiao Y, et al. Scheduling Cluster Tools in Semiconductor Manufacturing: Recent Advances and Challenges[J]. IEEE Transactions on Automation ence and Engineering, 2017:586-601.

[8] Ja-Hee Kim, Tae-Eog Lee, et al, Scheduling analysis of time-constrained dual-armed cluster tools[J]. IEEE Transactions on Semiconductor Manufacturing, 2003:521-534. 